\title{
Confrontando Estruturas de Valores: Um Estudo Comparativo entre PVQ-40 e PVQ-21
}

\author{
Confronting Structures of Values: A Comparative Study \\ between $P V Q-40$ and $P V Q-21$
}

\author{
Marta Fabiano Sambiase ${ }^{*}, a$, Maria Luisa Mendes Teixeira ${ }^{a}$, Wolfgang Bilsky ${ }^{b}$, \\ Bruno Felix Von Borell de Araujo ${ }^{c} \&$ Silvia Marcia Russi De Domenico ${ }^{a}$ \\ ${ }^{a}$ Universidade Presbiteriana Mackenzie, São Paulo, SP, Brasil, \\ ${ }^{b}$ University of Muenster, Muenster, Germany \\ $\&^{c}$ Fucape - Fundação Instituto Capixaba de Pesquisa em Contabilidade, Economia e Finanças, Vitória, ES, Brasil
}

\begin{abstract}
Resumo
Esforços têm sido realizados para desenvolver escalas de mensuração capazes de traduzir o constructo e a estrutura de valores. Entre as existentes estão as escalas SVS - Schwartz Value Survey e o Portrait Values Questionnaire com 40 e 21 itens (respectivamente PVQ-40 e PVQ-21). As duas formas desse último questionário apresentam aplicabilidade mais ampla, por possuírem uma menor quantidade de itens e terem os itens redigidos de forma menos abstrata do que a SVS. Não foram encontrados, no entanto, estudos que comparem as duas escalas quanto à estrutura de valores obtida a partir de sua aplicação, objetivo a que este estudo se propôs a alcançar. A pesquisa foi realizada com uma amostra de 217 estudantes universitários, aos quais foram aplicadas as escalas PVQ-40 e PVQ-21, em dois momentos distintos. Os dados foram tratados mediante Análise de Escalonamento Multidimensional Confirmatória Fraca. Os resultados indicaram que ambas as escalas são equivalentes quanto à estrutura de valores bidimensional e bipolar, porém foram encontradas divergências entre ambas quanto à distribuição no espaço dos valores dos tipos Estimulação, Autodeterminação, Poder e Realização. Palavras-chave: Escala, valores básicos, PVQ-40, PVQ-21, Análise de Escalonamento Multidimensional Confirmatória Fraca.
\end{abstract}

\begin{abstract}
Several measurement scales have been developed in order to translate constructs and their value structure. Among these are the Schwartz's Values Survey (SVS) and the Portrait Values Questionnaire with 40 and 21 items, respectively (PVQ-40 and PVQ-21). The two forms of the questionnaire allow a wider use because they have showed fewer and less abstract items compared to the SVS, however, there have not been found studies that compare both instruments regarding their value structure. Therefore, this is the objective of the present study. A sample of 217 university students answered the PVQ-40 and the PVQ-21 at two different moments in time. The data were analyzed by Confirmatory Multidimensional Scaling. The results indicate that both scales are equivalent in terms of bidimensional and bipolar value structures, however, differences were found with regard to the spatial distribution of values measuring Stimulation, Self-Direction, Power and Achievement. Keywords: Scale, basic values, PVQ-40, PVQ-21, Confirmatory Multidimensional Scaling.
\end{abstract}

Os estudos sobre valores humanos têm oferecido importantes contribuições desde Allport, no início do século passado e, mais notadamente, a partir da elaboração da teoria de valores básicos de Shalom Schwartz na passagem dos anos 1980 para os anos 90 (Schwartz, 1992; Schwartz \& Bilsky, 1987), cuja consistência vem sendo comprovada em amostras de diferentes culturas (Schwartz, 2005a, 2005b, 2006a).

\footnotetext{
* Endereço para correspondência: Centro de Ciências Sociais e Aplicadas, Universidade Presbiteriana Mackenzie, Rua da Consolação, 930, Predio 45, Secretaria CCSA, Consolação, São Paulo, SP, Brasil 01302-907. E-mail: martafsambiase@gmail.com
}

A existência de valores universais é uma premissa adotada desde os filósofos da Axiologia até os pesquisadores da psicologia social com foco no indivíduo. As diferenças de valores entre os homens ocorreriam pela prioridade que lhes fosse atribuída individualmente.

Rokeach (1973) assim considerando, propôs uma relação de 36 valores que, em 1967, passou a compor um inventário dividido em duas listas, visando à identificação dos sistemas de valores terminais e instrumentais dos indivíduos. O Rokeach Value Survey (RVS) é um dos mais conhecidos e utilizados instrumentos nas pesquisas de valores, tendo sido substituído nas últimas duas décadas pelo SVS (Schwartz Value Survey), elaborado por Schwartz, à 
Sambiase, M. F., Teixeira, M. L. M., Bilsky, W., Araujo, B. F. V. B. \& De Domenico, S. M. R. (2014). Confrontando Estruturas de Valores: Um Estudo Comparativo entre PVQ-40 e PVQ-21.

luz de sua teoria de valores básicos. Considerado o inventário mais recomendado para avaliar as prioridades dos valores gerais em função de sua qualidade psicométrica e larga utilização em pesquisas transculturais, o SVS não é de rápida aplicação uma vez que pode ultrapassar 60 itens ao incorporar valores de culturas específicas, além de exigir elevado grau de abstração do respondente (Porto, 2005). Em busca de parcimônia, alguns pesquisadores produziram instrumentos, reduzindo o número de itens do SVS, todavia, sem atentarem para os princípios da teoria de Schwartz (Teixeira \& Monteiro, 2008).

O Portrait Values Questionnaire (PVQ) foi a resposta de Schwartz, não só à busca de um instrumento com menor quantidade de assertivas, mas que representasse a teoria de valores básicos que desenvolveu, viabilizando pesquisas com adolescentes e crianças e, com adultos de menor nível de escolaridade. Constituído por 40 itens em sua versão final, foi validado em diversos países, inclusive no Brasil (Porto, 2005). Mais recentemente, uma versão reduzida com 21 itens (PVQ-21) foi desenhada com o propósito de realizar pesquisas na Europa, em uma rede de pesquisadores de diferentes países denominada European Social Survey (ESS). Alguns estudos foram feitos utilizando-se a base de dados do ESS, mas ainda resta uma discussão sobre a ampla utilização do PVQ-21 (Schwartz, comunicação pessoal, 18 de outubro, 2008), propósito a que este artigo se dispôs a atender, ao ter como objetivo comparar as estruturas de valores pessoais obtidas a partir da aplicação das duas versões para o português do Brasil do Perfil de Valores Pessoais - PVQ-40 (ou PQ-40) e PVQ-21, oferecendo para isso, uma validação do PVQ21, a partir de uma amostra de estudantes universitários na cidade de São Paulo, em uma investigação de natureza descritivo-exploratória com tratamento de dados por estatística multivariada. Espera-se poder contribuir para auxiliar na escolha entre as escalas PVQ-40 e PVQ-21 de pesquisadores ou profissionais que desejarem aplicá-la, de acordo com os objetivos a serem atendidos.

\section{A Teoria de Valores Básicos de Schwartz}

Ao rever a literatura, Schwartz (2006a) identificou cinco características dos valores: são crenças, transituacionais, sobre fins ou formas de comportamento desejáveis, que orientam a seleção e avaliação de comportamentos, pessoas e acontecimentos, organizados segundo a importância relativa que lhes é atribuída, resultando em um sistema de valores. Ainda que auxiliem a diferenciá-los de outros construtos, tais características não deixam claro qual é o conteúdo substantivo dos valores humanos capaz de classificá-los e relacioná-los.

Buscando avançar sobre a proposta de separação dos valores em terminais e instrumentais, apresentada por Rokeach (1973), Schwartz e Bilsky (1987) iniciaram o desenvolvimento de uma teoria de valores humanos básicos, questionando a referida classificação e propondo metas motivacionais como o conteúdo substantivo e organizador dos valores, em resposta a três necessidades inerentes a qualquer ser humano - biológicas, de interação social coordenada e de funcionamento e manutenção dos grupos, as quais são comunicadas mediante a linguagem dos valores (Schwartz, 2005a) e guiam os comportamentos de entidades sociais Schwartz (1992). Mesmo que homens e mulheres em sociedades distintas deem respostas diferenciadas a essas necessidades, dentro da especificidade de suas culturas, esses valores específicos estariam contemplados em dez categorias denominadas 'tipos motivacionais', ou valores básicos, que se diferenciam em função dos conteúdos motivacionais que representam. A análise desses conteúdos possibilitou identificar relações de compatibilidade e conflito entre os tipos motivacionais, resultando em uma estrutura, na qual os tipos adjacentes compartilham ênfases motivacionais, enquanto aqueles em oposição indicam conflito de metas, refletindo um continuum motivacional, que resultou em uma estrutura circular (Schwartz, 1992; Figura 1).

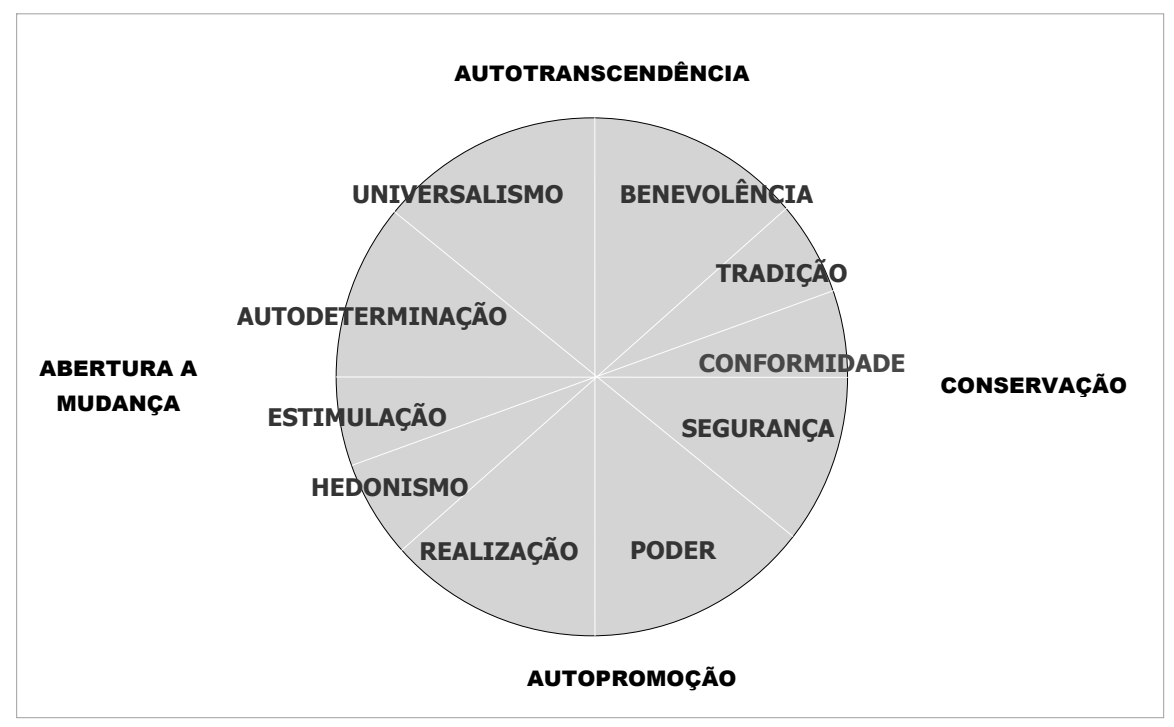

Figura 1. Estrutura teórica das relações entre tipos motivacionais. 
As compatibilidades e conflitos entre tipos motivacionais permitiu a identificação de duas dimensões bipolares - Autopromoção-Autotranscendência e Abertura a Mudança-Conservação (Schwartz, 1992). A primeira contrapõe metas relacionadas à busca do auto-interesse (Poder e Realização) a metas que refletem a preocupação pelos outros, que podem estar próximos (Benevolência) ou distantes (Universalismo); a segunda agrega os conteúdos motivacionais relacionados à busca do novo, variado e prazeroso (Autodeterminação, Estimulação e Hedonismo) em oposição à busca do que é tradicional, regrado e seguro (Tradição, Conformidade e Segurança). Mediante a aplicação do SVS, instrumento elaborado tendo como base a teoria de valores básicos, em pesquisa transcultural envolvendo inicialmente 44 países, inclusive o Brasil, espalhados nos cinco continentes, foi possível comprovar a quase universalidade dos dez tipos motivacionais e a universalidade das duas dimensões em oposição, comprovando a não-separação dos valores em terminais e instrumentais, conforme propunha Rokeach (1973).

\section{Mensuração de Valores Pessoais à Luz da Teoria de Valores Básicos}

Schwartz, para a elaboração do instrumento que testaria as hipóteses da teoria de valores, selecionou valores específicos que representassem cada uma das metas motivacionais ali propostas. Para isso, contou com listas de valores já utilizadas em instrumentos anteriores, principalmente o RVS de Rokeach, e outros como de Braithwaite e Law (1985), Hofstede (1984, citado em Schwartz, 1992). A análise de textos de religiões comparadas e consultas a pesquisadores islâmicos e drusos contribuíram com a adição de outros itens. Em sua primeira versão, o SVS compôs-se de 56 itens, divididos em duas relações que mantinham a premissa de Rokeach, da classificação dos valores em terminais e instrumentais, sendo os primeiros expressos como substantivos (30) e os segundos enquanto adjetivos (26) (Schwartz, 1992).

A escala SVS utilizada na mensuração dos valores é de nove pontos, numerada em 7 (suprema importância), 6 (muito importante), 5 e 4 (não rotulados), 3 (importante), 2 e 1 (não rotulados), 0 (não importante), -1 (oposto aos meus valores). Antes de responder aos itens, o respondente deve ler todos em cada uma das listas, escolhendo os itens de suprema importância e aqueles opostos a seus valores, em um processo denominado 'ancoramento', evitando o uso distorcido da escala (Schwartz, 2005a; Tamayo, 2007). Em 1994, Schwartz apresentou uma edição revisada do instrumento, com 57 itens, resultante da exclusão de um valor freqüentemente mal interpretado e incluindo outros dois (Schwartz, 2005a). Os índices de confiabilidade (alpha de Cronbach) médios encontrados para os dez tipos motivacionais levando-se em conta 212 amostras, variaram entre 0,60 (tradição) até 0,75 (universalismo) (Schwartz, 2005b).

Relativamente ao RVS, o SVS representa um avanço sob três aspectos (Tamayo, 2007). Primeiramente, por considerar uma relação mais representativa dos valores humanos, contemplando culturas que não apenas a americana. Em segundo lugar, cada valor, após o procedimento de ancoragem, é avaliado de forma independente (rating), o que permite a realização de análises estatísticas mais robustas do que a escala ordinal (ranking) proposta por Rokeach. Por último, apresenta uma estrutura de tipo fatorial e avalia valores de acordo com a proposta teórica de Schwartz, que abrange uma estrutura de relações dinâmicas entre os valores.

O SVS é tido como o instrumento mais utilizado para avaliar os 10 tipos motivacionais da teoria de valores básicos (Schwartz, 2005b) e foi traduzido para 47 línguas pelo processo de tradução-retradução (Schwartz, 2006a), incluindo-se o português do Brasil (Tamayo \& Schwartz, 1993). Apesar da ampla aceitação do instrumento na comunidade acadêmica e de outros teóricos confirmarem a adequação do SVS à teoria proposta por Schwartz (Gouveia, Martínez, Meira, \& Milfont, 2001), algumas preocupações levaram-no a desenvolver um instrumento adicional. Entre elas, resultados insatisfatórios em relação à estrutura de valores obtidos em aplicações do SVS em áreas rurais de países menos desenvolvidos e em sociedades com sistema educacional não-ocidental. Também o alto grau de abstração que o SVS exige do respondente dificultava a aplicação em amostras de jovens e adolescentes. Além dessas limitações, quanto mais instrumentos de medida de valores pessoais levassem a resultados comprovando a estrutura teórica de valores, mais sólida seria a teoria proposta (Schwartz, 2005b).

Tais reflexões levaram ao desenvolvimento de um novo instrumento: o PVQ, que mede os valores dos indivíduos mediante curtas descrições verbais. Inicialmente com 29 e, posteriormente, com 40 perfis de indivíduos, elaborados em duas frases consecutivas, o PVQ indica objetivos, aspirações ou desejos que apontam para cada um dos dez tipos motivacionais. O número de perfis depende do tipo motivacional considerado, levando-se em conta a amplitude das definições conceituais de cada um deles. Assim, para mensurar o tipo universalismo, foram elaboradas seis descrições, enquanto apenas três foram necessárias para estimulação, hedonismo e poder na escala de 40 itens (Schwartz, 2005b).

Para cada perfil apresentado, responde-se à seguinte pergunta: "Quanto essa pessoa se parece com você?" As cinco alternativas possíveis de resposta, oferecidas em uma escala perceptual, tipo Likert, são: se parece muito comigo, se parece comigo, se parece mais ou menos comigo, não se parece comigo, não se parece nada comigo.

Os valores dos sujeitos são identificados a partir da similaridade apontada entre o perfil descrito e o do respondente. Enquanto o SVS exige uma avaliação consciente de valores específicos, o PVQ mede-os indiretamente, implicitamente (Schwartz, 2005b; Tamayo, 2007). Aplicado inicialmente em amostras de adultos, universitários e adolescentes em diversos países da Europa, América do Sul e Indonésia, os resultados do PVQ apoiaram a estru- 
Sambiase, M. F., Teixeira, M. L. M., Bilsky, W., Araujo, B. F. V. B. \& De Domenico, S. M. R. (2014). Confrontando Estruturas de Valores: Um Estudo Comparativo entre PVQ-40 e PVQ-21.

tura teórica de relações entre os tipos motivacionais. Os índices de confiabilidade médios, usando-se amostras de 25 países diferentes, situam-se em 0,47 (tradição), 0,60 (autodeterminação), 0,66 (segurança), 0,67 (conformidade, benevolência), 0,68 (poder), 0,71 (estimulação), 0,77 (universalismo), 0,79 (hedonismo) e 0,80 (realização) (Schwartz, 2005b).

O PVQ foi traduzido e validado para o português do Brasil por Tamayo e seus colaboradores (Porto, 2005; Tamayo, 2007). Aplicado a uma amostra de 614 brasileiros, com grau de escolaridade inferior ao ensino médio, o PQ, como é chamada a versão brasileira para o questionário de 40 itens, levou a resultados que apóiam a teoria de valores básicos, que também prevê agrupamentos de tipos motivacionais adjacentes. Assim, no Brasil, apenas universalismo, benevolência, segurança e auto-direção formaram regiões diferenciadas, enquanto comparecem em regiões conjuntas, dois a dois, conformidade e tradição, poder e realização, hedonismo e estimulação (Tamayo \& Porto, 2009). Apesar de chamar atenção para a necessidade de aprimoramento do PQ, Porto (2005) ressalta o aspecto promissor do uso dessa escala para a avaliação de propriedades axiológicas, verificando-se que a mesma tem sido empregada não só por pesquisadores do Brasil (ex.: Kamia, 2007; Silva, 2010), mas também do exterior (ex.: Chang \& Lin, 2008).

Em busca de instrumentos mais parcimoniosos, uma nova versão (PVQ-21) foi desenhada com quase a metade de itens, para atender aos requisitos de uma pesquisa on line (Schwartz, 2006b). Realizada pela European Social Survey e conduzida em mais de 30 nações mediante coleta de dados em prazos bienais desde 2002 (ESS, 2009), os dados coletados têm servido para diversos pesquisadores realizarem seus estudos, tais como Baslevent e Kirmanoglu (2008), que avaliaram o papel dos valores pessoais nas opções de voto em eleições na Turquia, enquanto Davidov, Schmidt e Schwartz (2008) empregaram as mensurações de valores fornecidas pela ESS em estudo transcultural a respeito dos efeitos destes na atitude quanto à imigração.

O PVQ-21 segue as mesmas características contempladas no PVQ-40, com a distinção de conter 21 itens retirados da versão original. Alguns foram revisados com a preocupação de abranger o máximo do conteúdo motivacional de cada um dos dez valores básicos (Schwartz, 2006a). Cada tipo motivacional ficou representado por dois itens, exceto universalismo, que conta com três, o que reduziu os índices de confiabilidade (alpha de Cronbach) no que diz respeito aos dez tipos motivacionais, variando entre 0,36 (tradição) e 0,70 (realização).

Não obstante Schwartz (2006a) reconheça que os valores básicos mensurados pelo PVQ-21 predizem atitudes e comportamento sistematicamente, desaconselha sua utilização (Schwartz, comunicação pessoal, 18 de outubro, 2008), preferindo a versão original (PVQ-40), uma vez que o primeiro possui menos itens que o esperado para uma medida confiável. Além disso, se o PVQ-21 é preenchido em cinco minutos, o segundo demanda apenas oito minutos do respondente, considerando três minutos uma diferença muito pequena para justificar a preferência pela versão reduzida em aplicações outras que não a pesquisa ESS.

De fato, todos os artigos identificados na revisão da literatura empregando o PVQ-21, utilizaram o banco de dados disponível no ESS (2009). Esforços têm sido feitos para analisar a escala em si, como o estudo de Verkasalo, Lönnqvist, Lipsanen e Helkama (2008) que propõem o colapso dos dez tipos nos quatro pólos das dimensões bipolares - estes sim, com alta confiabilidade.

Também Davidov, Meuleman, Billiet e Schmidt (2008) testaram os dados obtidos com o PVQ-21 em amostras de 20 países provenientes da ESS, obtendo a invariância de configuração e métrica somente após unificar pares de valores que apresentavam alta correlação. Ao final, foram identificados sete valores: segurança, autodeterminação, estimulação, hedonismo e as combinações entre tradição/ conformidade, universalismo/ benevolência e poder/ realização, sugerindo que a versão de 21 itens é capaz de contemplar o círculo motivacional de valores da teoria no qual está embasada.

Como sugere Schwartz (comunicação pessoal, 18 de outubro, 2008) faz-se necessária a ampliação da pesquisa, comparando as duas escalas quanto ao seu desempenho, tendo em vista a teoria dos valores básicos por ele proposta. No Brasil, além de ainda não haver sido realizada pesquisa comparando o desempenho das duas escalas, também não foi apresentada a validação da escala PVQ de 21 itens, objetivos que este artigo buscou alcançar.

\section{Procedimentos Metodológicos}

Para responder ao objetivo principal deste estudo, cuja natureza é eminentemente metodológica, e que é comparar as estruturas de valores pessoais obtidas a partir da aplicação das duas versões do Perfil de Valores Pessoais PVQ-40 (ou PQ-40) e PVQ-21, foi realizada uma pesquisa de tipo descritiva, quantitativa. O objetivo principal deste tipo de pesquisa é a descrição de algo, ao mesmo tempo em que procura relatar como ocorrem certos fenômenos, explica Malhorta (2001). Optou-se por um survey, com os dados tratados em dois momentos de uma amostra também coletada em duas situações distintas com os mesmos respondentes (Babbie, 1999). A seguir é detalhada a origem e tradução dos instrumentos de coleta de dados, coleta, codificação, tratamento dos dados e análise dos resultados.

\section{Instrumento de Coleta de Dados}

Para a aplicação do PVQ-40 nesta pesquisa, foi utilizada uma versão da mesma já traduzida para o português (PQ-40). A versão do PVQ-21 em inglês foi extraída do ESS (2009) e esta foi encaminhada para tradução para o português do Brasil por três pesquisadores separadamente; estes pesquisadores foram escolhidos por conhecerem o PVQ-40 e terem prática no estudo de valores básicos no ambiente brasileiro. Também foi solicitada uma tradução profissional do PVQ-21 para a empresa BTSGlobal, que 
utiliza tradutores profissionais sem conhecimento do instrumento de coleta de valores básicos. Com as quatro traduções finalizadas, estas foram colocadas em paralelo e, diante do PVQ-21 original, solicitando-se a dois outros pesquisadores, que apontassem as frases traduzidas que melhor retratavam a assertiva original. Por fim, o PVQ-21, traduzido e utilizado em Portugal, foi analisado e as assertivas comparadas com o PVQ-21 em português brasileiro; mesmo a língua portuguesa sendo diferente do português do Brasil, comparou-se o sentido das frases para garantir maior confiabilidade do processo de tradução.

Foi realizado um pré-teste com trinta alunos de curso de graduação, mediante a aplicação dos dois questionários objetos de análise deste estudo; como não houve dúvidas para responder as assertivas, nem problemas de interpretação, as mesmas foram mantidas para a aplicação em maior quantidade e comparecem na Figura 2.

\begin{tabular}{|c|}
\hline PVQ 21 (ESS, 2009) \\
\hline $\begin{array}{l}\text { 1) Pensar em novas idéias e ser criativa é importante para ela. Ela gosta de fazer as cois as de maneira } \\
\text { própria e original. }\end{array}$ \\
\hline 2) Ser rica é importante para ela. Ela quer ter muito dinheiro e possuir cois as caras. \\
\hline $\begin{array}{l}\text { 3) Ela acredita que é importante que todas as pes soas do mundo sejam tratadas igualmente. Ela acredita } \\
\text { que todos deveriam ter oportunidades iguais na vida. }\end{array}$ \\
\hline 4) É muito importante para ela demonstrar suas habilidades. Ela quer que as pessoas admirem o que ela faz. \\
\hline $\begin{array}{l}\text { 5) É importante para ela viver em um ambiente seguro. Ela evita qualquer coisa que pos sa colocar sua } \\
\text { segurança em perigo. }\end{array}$ \\
\hline $\begin{array}{l}\text { 6) Ela gosta de surpresas e está sempre procurando cois as novas para fazer. Ela acha ser importante fazer } \\
\text { muitas cois as diferentes na vida. }\end{array}$ \\
\hline $\begin{array}{l}\text { 7) Ela acredita que as pessoas deveriam fazer o que lhes é ordenado. Ela acredita que as pessoas deveriam } \\
\text { sempre seguir as regras, mesmo quando ninguém es tá observando. }\end{array}$ \\
\hline $\begin{array}{l}\text { 8) É importante para ela ouvir as pessoas que são diferentes dela. Mesmo quando não concorda com elas, } \\
\text { ainda quer entende-las. }\end{array}$ \\
\hline 9) É importante para ela ser humilde e modesta. Ela tenta não chamar atenção para si. \\
\hline 10) Aproveitar os prazeres da vida é importante para ela. Ela gosta de se mimar. \\
\hline $\begin{array}{l}\text { 11) É importante para ela tomar suas próprias decisões sobre o que faz. Ela gosta de ser livre e não } \\
\text { depender dos outros. }\end{array}$ \\
\hline 12) É muito importante para ela ajudar as pessoas ao seu redor. Ela quer cuidar do bem-estar delas. \\
\hline 13) Ser muito bem-sucedida é importante para ela. Ela espera que as pessoas reconheçam suas realizações. \\
\hline $\begin{array}{l}\text { 14) É importante para ela que o governo garanta sua segurança contra todas as ameaças. Ela des eja que o } \\
\text { Estado s eja forte para poder defender seus cidadãos. }\end{array}$ \\
\hline 15) Ela procura por aventuras e gosta de correr riscos. Ela quer ter uma vida excitante. \\
\hline $\begin{array}{l}\text { 16) É importante para ela sempre se comportar de modo adequado. Ela quer evitar fazer qualquer coisa que } \\
\text { as pessoas possam dizer que é errado. }\end{array}$ \\
\hline 17) É importante para ela ter o respeito dos outros. Ela deseja que as pessoas façam o que ela diz. \\
\hline 18) É importante para ela ser leal a seus amigos. Ela quer se dedicar às pessoas próximas a ela. \\
\hline $\begin{array}{l}\text { 19) Ela acredita firmemente que as pessoas deveriam pres ervar a natureza. Cuidar do meio ambiente é } \\
\text { importante para ela. }\end{array}$ \\
\hline $\begin{array}{l}\text { 20) Tradição é importante para ela. Ela procura seguir os costumes transmitidos por sua religião ou pela } \\
\text { sua família. }\end{array}$ \\
\hline $\begin{array}{l}\text { 21) Ela procura todas as oportunidades para se divertir. É importante para ela fazer cois as que lhe tragam } \\
\text { prazer. }\end{array}$ \\
\hline
\end{tabular}

Figura 2. Assertivas do PVQ-21 em português do Brasil.

\section{Unidade de Análise, População, Sujeito de Pesquisa, Amostra}

A unidade de análise da pesquisa é o indivíduo, por se tratar de valores básicos. O sujeito de pesquisa foi o aluno de cursos de graduação de Administração de Empresas e a população composta por alunos desse curso de duas universidades localizadas na cidade de São Paulo. O objetivo amostral foi definido em função da quantidade mínima indicada para procedimentos confirmatórios de escala, que são duzentos casos (Hair, Anderson, \& Black, 2005).
A amostra não probabilística inicial foi composta por 217 respondentes. A aplicação deu-se mediante a distribuição de questionários em papel na sala de aula em duas etapas com intervalo de uma semana: o PVQ-40 primeiramente e, o PVQ-21, aplicado aos mesmos alunos após uma semana. O intervalo entre a aplicação de um questionário e outro foi necessário para evitar que os alunos repetissem a resposta do PVQ-40 no PVQ-21.

Para efeito da comparação das duas escalas foi realizada a análise Escalanomento Multidimensional Confirmatória 
Sambiase, M. F., Teixeira, M. L. M., Bilsky, W., Araujo, B. F. V. B. \& De Domenico, S. M. R. (2014). Confrontando Estruturas de Valores: Um Estudo Comparativo entre PVQ-40 e PVQ-21.

(MDS Confirmatória), conforme sugere Bilsky, Gollan e Döring (2008), Bilsky e Janik (2010), Borg, Groenen, Jehn, Bilsky e Schwartz (2011), denominada por Borg, Groenen, e Mair (2013), de weak Confirmatory MDS, para a qual foi adotada neste trabalho a sigla WCMDS. Para tanto foi necessário escolher entre a estrutura original proposta por Schwartz (1992) e a revisada conforme Schwartz e Sagiv (1995). No estudo realizado por Bilsky e Janik (2010), a estrutura de valores é compatível tanto com o modelo original, quanto com o modelo revisado. Em Schwartz e Sagiv (1995), os valores Tradição e Conformidade compartilham o mesmo espaço angular, resultando num círculo dividido em nove regiões, em vez de dez, como na estrutura original, em que os valores "Tradição" e "Conformidade" ocupam espaços adjacentes. De acordo com os autores a configuração original foi considerada a melhor por ser mais simples, parcimoniosa e mais adequada para tratamentos mais complexos como, por exemplo, MM- Multimétodo Multitraço. Por esta razão o modelo escolhido para orientar a análise WCMDS realizada neste artigo foi o modelo original de Schwartz (1992).

A análise WCMDS foi realizada considerando a matriz de desenho proposta por Bilsky, Gollan, et al. (2008, p. 215), onde as "coordenadas correspondem aos centros dos arcos determinados pelos distintos setores de valores", modificada uma vez que aquela estava adequada ao modelo revisado de Schwartz e Sagiv (1995), e o modelo escolhido para esta pesquisa foi o modelo original de Schwartz (1992).

De acordo com Bilsky e Janik (2010), apesar de autores como Borg e Staufenbiel (2007) argumentarem apontando o fraco poder confirmatório da MDS, esta é preferível ao emprego da MDS exploratória, sendo as matrizes de desenho propostas por Bilsky, Gollan, et al. (2008), o primeiro passo para a realização da tarefa.

Trabalhos empregando a WCMDS são raros, sendo exceções os realizados por Borg et al. (2011), conforme mencionado por Bilsky e Janik (2010). Assim sendo a relevância deste artigo destaca-se não só pelos objetivos que se propôs a alcançar, mas também pelos procedimentos metodológicos adotados.

Rosenberg e Kim (1975) explicam que o Multidimensional Scaling (MDS) - Proxscal é um tratamento que permite montar a estrutura de medidas de proximidade em um conjunto de objetos. Este processo é realizado através da atribuição de observações em locais específicos num espaço conceitual multidimensional, de tal forma que as distâncias entre os pontos no espaço coincidem com as semelhanças dos dados, neste caso, tanto quanto possível. O tratamento de dados foi feito com os softwares estatísticos Excel 2007 e SPSS v. 15 e os resultados são apresentados na próxima sessão.

\section{Resultados}

\section{Preparação e Qualidade dos Dados da Amostra}

O ESS (2009, Capítulo 4, p. 3) recomenda um percentual aceitável de dados faltantes de $23,8 \%$ e $76,2 \%$ para a repetição de respostas, relativo ao PVQ-21. O mesmo critério foi utilizado para o PVQ-40 por ser a orientação mais recente e mais rigorosa, priorizando a qualidade dos dados. Assim, procedeu-se a eliminação e substituição de casos.

Das 217 respostas iniciais de ambos os questionários, 5 registros foram extraídos por terem ultrapassado o limite aceitável de dados faltantes, resultando em uma amostra final de 212 casos. Destes, 16 registros tinham campos faltantes dentro do limite aceitável, os quais foram preenchidos com a média das respostas das questões (Hair et al., 2005).

Outro procedimento aconselhável (ESS, 2009) para facilitar o tratamento dos dados é a inversão da escala tipo Likert. Em ambos os questionários de valores, esta inicia com o número 1 no comportamento "se parece muito comigo" até 6 "não se parece nada comigo". O comando "recover" do software SPSS foi utilizado para inverter a ordem para que a escala numérica crescente represente o comportamento também crescente.

Para efeito de considerar a adequação dos dados para a realização de WCMDS foram analisados os níveis de stress, os quais ficaram dentro da margem de 0,20 , conforme sugere Schwartz (1992). Na Figura 3 é apresentada a lógica utilizada para a codificação das variáveis que estão no tratamento de dados e figuras ao longo dos resultados. Validação da Estrutura do PVQ-40

A análise WCMDS da escala PVQ-40, considerando os 40 itens (Figuras 4 e 5), mostra que a mesma reflete a estrutura do modelo original de Schwartz (1992; Figura 1), porém com o espaço dividido em apenas oito regiões (Figura 5), tendo-se reunido na mesma região os valores de Universalismo (mUN) e Benevolência (mBE); Estimulação $(\mathrm{mST})$ e Autodeterminação $(\mathrm{mSD})$. Os valores relativos a Poder (mPO) e Realização (mAC) apresentaram posição trocada em relação ao modelo original, tendo Poder ficado localizado em adjacência a Hedonismo (mHE), enquanto Realização (mAC) localizou-se adjacente a Segurança (mSE). Esta troca já foi verificada em estudo realizado com a escala SVS aplicada a 168 estudantes mexicanos (Bilsky \& Peters, 1999), assim como em outro estudo realizado com a escala SVS e CPVC (Computerized Paired Comparison of Values) com estudantes alemães, a mesma troca de posições foi verificada (Bilsky, Brocke, \& Gollan, 2008).

Estes resultados diferem dos mencionados por Porto (2005), uma vez que os valores encontrados agrupados formando a mesma região não coincidem. Por outro lado os valores Universalismo e Benevolência que haviam configurado regiões distintas, nesta pesquisa, formaram uma única região. É interessante notar, no entanto, observando as Figura 4 e 5 que os mesmos aparecem em posição invertida, o que foi verificado em outras amostras conforme indica Schwartz (2005a). Os valores de Hedonismo e Estimulação, que segundo Porto (2005) haviam se reunido numa única região, no estudo que menciona, nesta pesquisa apareceram formando regiões distintas e em contrapartida foram os valores de Estimulação e Autodeterminação que se reuniram em uma única região. 


\begin{tabular}{|c|c|c|c|c|c|}
\hline \multicolumn{2}{|c|}{$\begin{array}{l}\text { Lógica para a Legenda de } \\
\text { Codificação das Variáveis }\end{array}$} & \multicolumn{2}{|c|}{$\begin{array}{l}\text { Lógica para a Legenda de } \\
\text { Codificação das Variáveis }\end{array}$} & \multicolumn{2}{|c|}{$\begin{array}{l}\text { Lógica para a Legenda de } \\
\text { Codificação das Variáveis }\end{array}$} \\
\hline vA1_21 & vP2_21 & vA1_40 & vP2_40 & mUN_21 & mAC_40 \\
\hline $\mathbf{v}=$ valor & $\mathbf{v}=$ valor & $\mathbf{v}=$ valor & $\mathbf{v}=$ valor & $\begin{array}{l}\mathbf{m}=\text { média do } \\
\text { Tipo Motiv. }\end{array}$ & $\begin{array}{l}\mathbf{m}=\text { média do } \\
\text { Tipo Motiv. }\end{array}$ \\
\hline $\begin{array}{c}\mathbf{A}=\text { Tipo Motiv. } \\
\text { (Autodetermin } \\
\text { ação) }\end{array}$ & $\begin{array}{c}\mathbf{P}=\text { Tipo Motiv } \\
\text { (Poder) }\end{array}$ & $\begin{array}{c}\mathbf{A}=\text { Tipo Motiv. } \\
\text { (Autodetermina } \\
\text { ção) }\end{array}$ & $\begin{aligned} \mathbf{P}= & \text { Tipo Motiv } \\
& \text { (Poder) }\end{aligned}$ & $\begin{array}{c}\mathbf{U N}=\text { Tipo } \\
\text { Motivacional } \\
\text { (em ingles) }\end{array}$ & $\begin{array}{c}\mathbf{A C}= \\
\text { Achievement } \\
(\text { em ingles })\end{array}$ \\
\hline $\begin{array}{c}\mathbf{1}=\text { nro questão } \\
\text { no PVQ }\end{array}$ & $\begin{array}{c}\mathbf{2}=\text { nro questão } \\
\text { no PVQ }\end{array}$ & $\begin{array}{c}\mathbf{1}=\text { nro questão } \\
\text { no PVQ }\end{array}$ & $\begin{array}{c}\mathbf{2}=\text { nro questão } \\
\text { no PVQ }\end{array}$ & 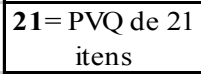 & $\begin{aligned} & \mathbf{4 0}= \text { PVQ de } 40 \\
& \text { itens }\end{aligned}$ \\
\hline $\begin{array}{c}\mathbf{2 1}= \\
\text { PVQ de } 21 \\
\text { itens }\end{array}$ & $\begin{array}{c}\mathbf{2 1}=\begin{array}{c}\text { PVQ de } 21 \\
\text { itens }\end{array} \\
\end{array}$ & \begin{tabular}{|c|}
$\mathbf{4 0}=$ \\
PVQ de 40 \\
itens
\end{tabular} & $\begin{array}{c}\mathbf{4 0}=\begin{array}{l}\text { PVQ de } 40 \\
\text { itens }\end{array} \\
\end{array}$ & \multicolumn{2}{|c|}{$\mathrm{UN}=$ Universalis $\mathrm{m}$} \\
\hline \multicolumn{2}{|c|}{$\mathrm{U}=$ Universalismo } & \multicolumn{2}{|c|}{$\mathrm{U}=$ Universalismo } & \multicolumn{2}{|c|}{$\mathrm{BE}=$ Benevolence } \\
\hline \multicolumn{2}{|c|}{$\mathrm{B}=$ Benevolencia } & \multicolumn{2}{|c|}{$\mathrm{B}=$ Benevolencia } & \multicolumn{2}{|c|}{$\mathrm{TR}=$ Tradiction } \\
\hline \multicolumn{2}{|c|}{$\mathrm{T}=$ Tradição } & \multicolumn{2}{|c|}{$\mathrm{T}=$ Tradição } & \multicolumn{2}{|c|}{$\mathrm{CO}=$ Conformity } \\
\hline \multicolumn{2}{|c|}{$\mathrm{C}=$ Conformidade } & \multicolumn{2}{|c|}{$\mathrm{C}=$ Conformidade } & \multicolumn{2}{|c|}{$\mathrm{SE}=$ Security } \\
\hline \multicolumn{2}{|c|}{$\mathrm{S}=$ Segurança } & \multicolumn{2}{|c|}{$\mathrm{S}=$ Segurança } & \multicolumn{2}{|c|}{$\mathrm{PO}=$ Power } \\
\hline \multicolumn{2}{|c|}{$\mathrm{P}=$ Poder } & \multicolumn{2}{|c|}{$\mathrm{P}=$ Poder } & \multicolumn{2}{|c|}{$\mathrm{AC}=$ Achievement } \\
\hline \multicolumn{2}{|c|}{$\mathrm{R}=$ Realização } & \multicolumn{2}{|c|}{$\mathrm{R}=$ Realização } & \multicolumn{2}{|c|}{$\mathrm{HE}=$ Hedonis $\mathrm{m}$} \\
\hline \multicolumn{2}{|c|}{$\mathrm{H}=$ Hedonismo } & \multicolumn{2}{|c|}{$\mathrm{H}=$ Hedonis mo } & \multicolumn{2}{|c|}{$\mathrm{ST}=$ Stimulation } \\
\hline \multicolumn{2}{|c|}{$\mathrm{E}=$ Estimulação } & \multicolumn{2}{|c|}{$\mathrm{E}=$ Estimulação } & \multicolumn{2}{|c|}{$\mathrm{SD}=$ Self Direction } \\
\hline \multicolumn{2}{|c|}{$\mathrm{A}=$ Autodeterminação } & \multicolumn{2}{|c|}{$\mathrm{A}=$ Autodeterminação } & & \\
\hline
\end{tabular}

Figura 3. Lógica para codificação das variáveis do tratamento de dados.

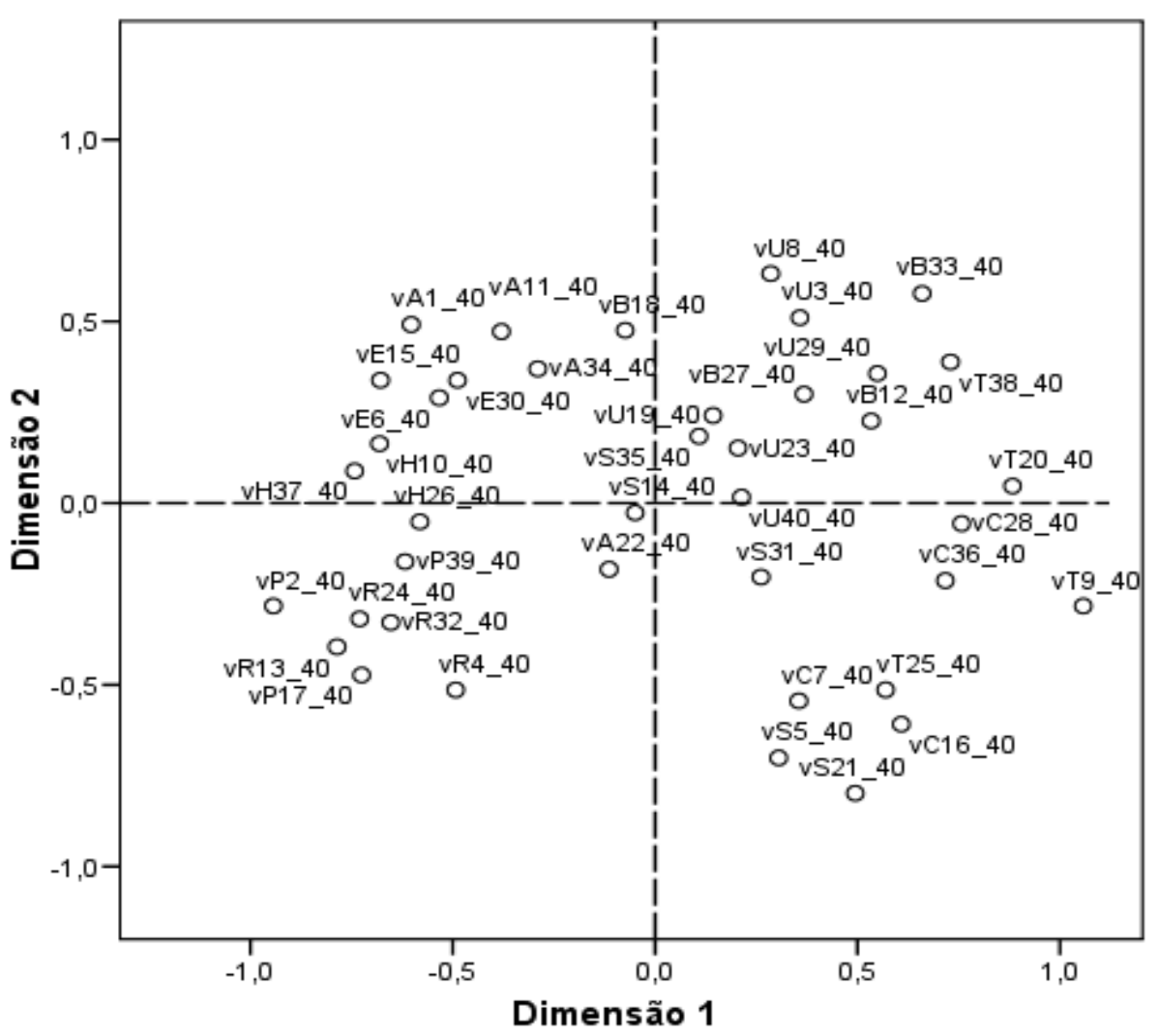

Figura 4. WCMDS por itens do PVQ40. 
Sambiase, M. F., Teixeira, M. L. M., Bilsky, W., Araujo, B. F. V. B. \& De Domenico, S. M. R. (2014). Confrontando Estruturas de Valores: Um Estudo Comparativo entre PVQ-40 e PVQ-21.

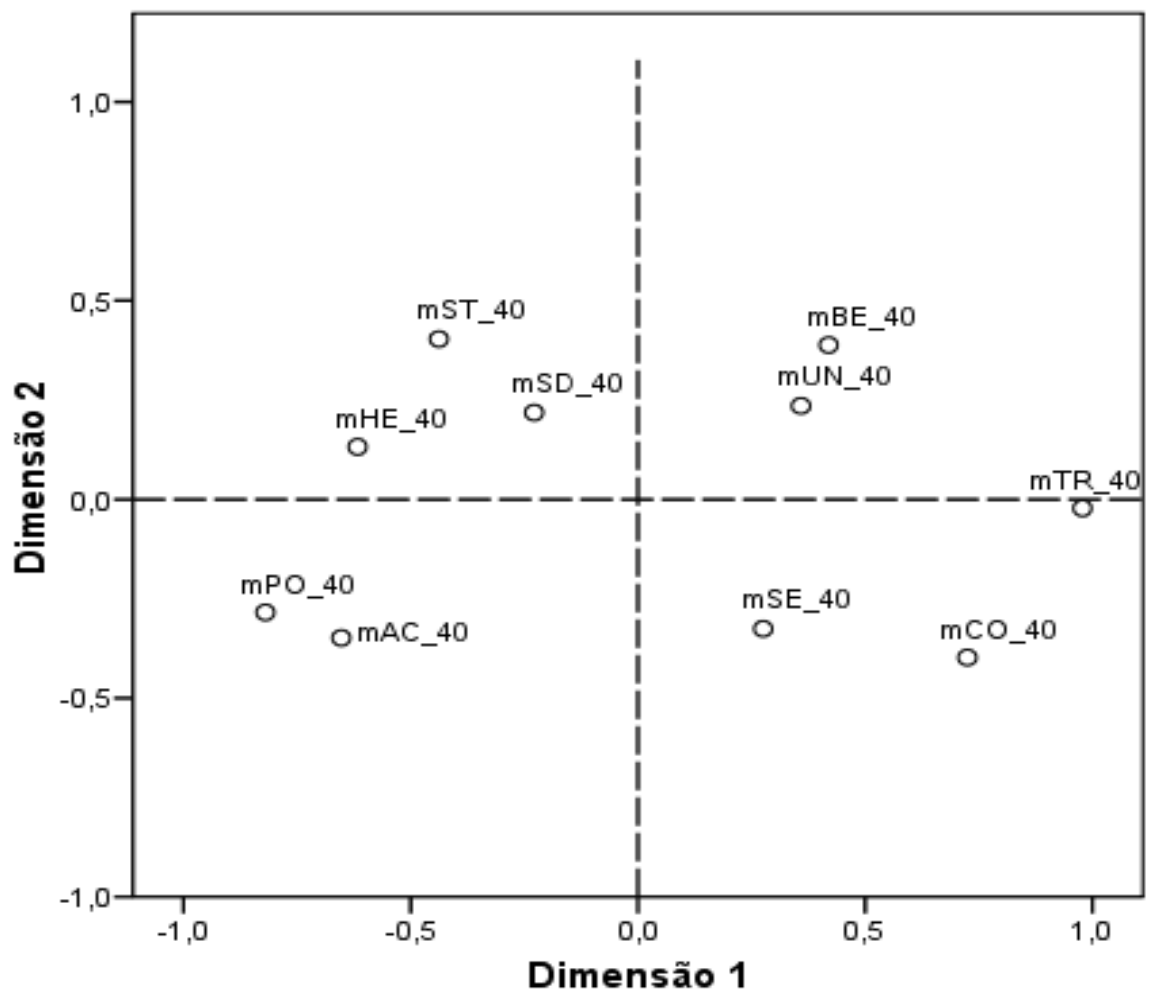

Figura 5. WCMDS por tipo motivacional - PVQ-40.

Porém, tanto o trabalho referido por Porto (2005), quanto os resultados neste trabalho, mostram que as regiões relativas às dimensões bipolares Autotranscendência versus Autopromoção, assim como Conservação versus Abertura a Mudança, foram corretamente posicionadas, corroborando a teoria de valores básicos de Schwartz (1992).

A confiabilidade dos itens variou de 0,425 - o menor índice para os valores relativos a Tradição, a $0,761-o$ maior índice - para Realização. Os valores Universalismo, Realização, Hedonismo, Estimulação e Poder obtiveram índices iguais ou superiores a 0,700; Conformidade e Segurança, acima de 0,600; e Benevolência e Autodeterminação com índices superiores a 0,500 . As dimensões por sua vez obtiveram índices de alpha de Cronbach variando entre 0,711 (Conservação) a 0,837 (Autopromoção).

Os resultados coincidem com os valores obtidos por Tamayo e Porto (2009) no que se refere ao valor Tradição, tendo sido este o que obteve o menor índice de confiabilidade $(0,47)$ e quanto ao maior índice obtido relativo aos valores de Realização $(0,80)$, a coincidência fica por conta do estudo realizado por Schwartz (2005b).
Validação da Estrutura do PVQ-21

Os resultados encontrados mostram que a escala PVQ-21 reflete a teoria de valores básicos de Schwartz (1992), de acordo com o modelo original, uma vez que as dimensões bi-polares de Autotranscendência versus Autopromoção, assim como a dimensão de Conservação versus Abertura a Mudança estão distribuídas corretamente no espaço (Figuras 6 e 7). Diferentemente do resultado obtido pela escala PVQ-40, os valores de Estimulação e Autodeterminação ocuparam posições distintas, enquanto que os valores de Poder e Realização ocuparam uma mesma região. Também a escala PVQ-21 revelou como a PVQ-40 que os valores de Universalismo e Benevolência ocupam uma mesma região. Estes dados condizem parcialmente com os encontrados por Davidov, Meuleman, et al. (2008) quanto às duas regiões comuns encontradas, porém a região única compreendida pelos valores de autodeterminação e estimulação encontrada nas amostras dos vinte países estudadas por esses autores, nesta pesquisa foram localizadas em espaços distintos. 


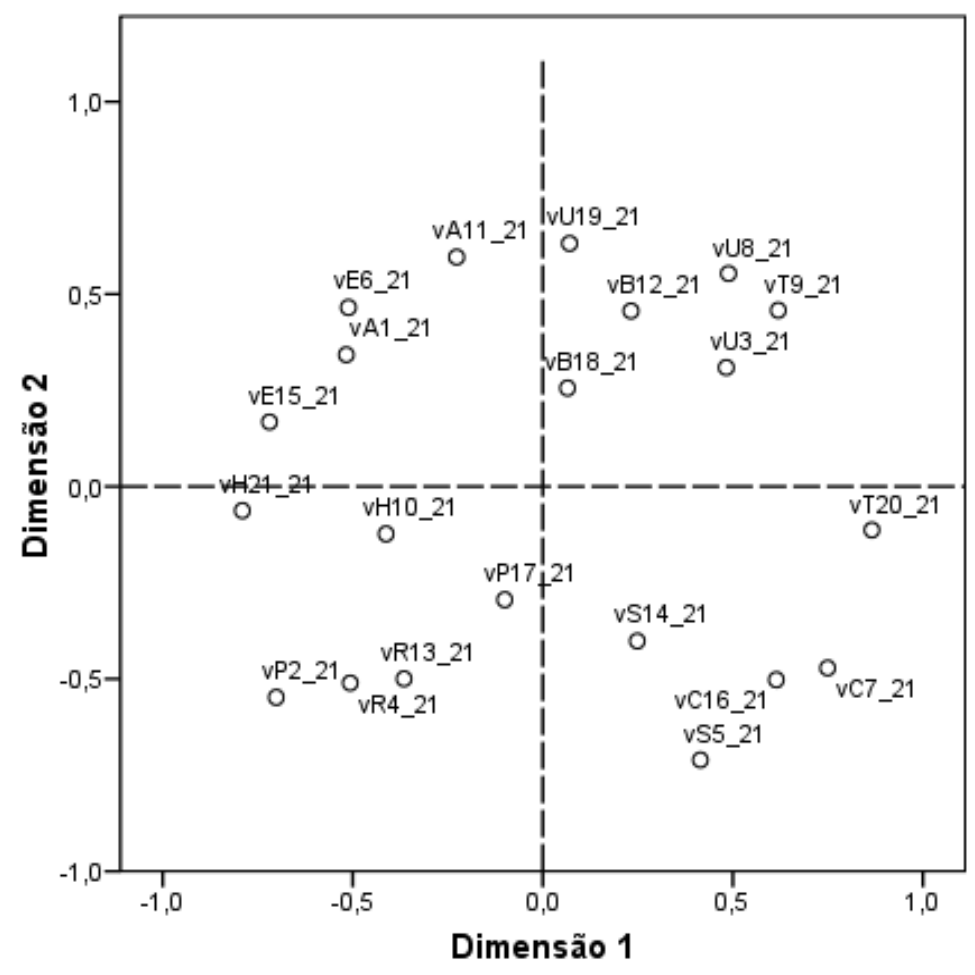

Figura 6. WCMDS por itens do PVQ-21

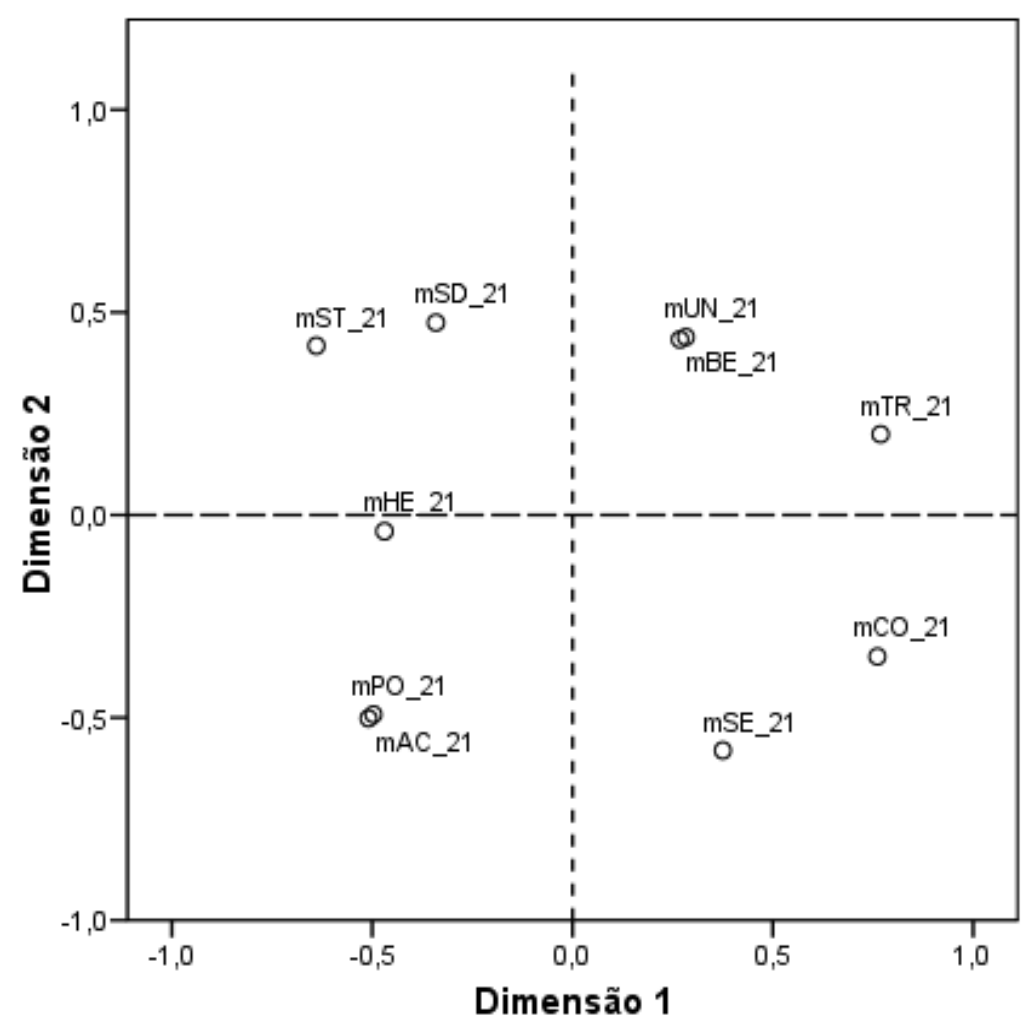

Figura 7. WCMDS por tipo motivacional - PVQ-21. 
Sambiase, M. F., Teixeira, M. L. M., Bilsky, W., Araujo, B. F. V. B. \& De Domenico, S. M. R. (2014). Confrontando Estruturas de Valores: Um Estudo Comparativo entre PVQ-40 e PVQ-21.

A confiabilidade calculada por meio do alpha de Cronbach mostrou que os índices mais elevados foram obtidos pelos valores de Benevolência $(0,614)$ e Realização $(0,646)$ e os mais baixos foram obtidos pelos valores de Tradição, Poder e Universalismo, estes com índices em torno de 0,300 , tendo os demais variado entre 0,450 e 0,560 . Em contrapartida, como era esperado, os índices relativos às dimensões tiveram variação menor entre si, situando-se em torno de 0,600.

\section{Confrontando as Estruturas das Escalas PVQ-40 e PVQ-21}

Analisando-se os resultados obtidos ao se reunirem as duas escalas e tratá-las mediante WCMDS a partir da matriz de desenho relativa ao modelo original de Schwartz (1992), verifica-se a perfeita compatibilidade entre ambas no que se refere às dimensões bipolares (Figura 8). Os valores de Poder (mPO) e Realização (mAC) de ambas as escalas configuram uma única região de Autopromoção, da mesma forma que os valores de Universalismo (mUN) e Benevolência (bem), em oposição, configuram a região de Autotranscendência. Os valores de Hedonismo (mHE), Estimulação (mST) e Autodeterminação (mAD) formam Abertura a Mudança, enquanto os valores de Tradição (mTR), Conformidade (com) e Segurança (mSE) reúnem-se em oposição na região de Conservação.

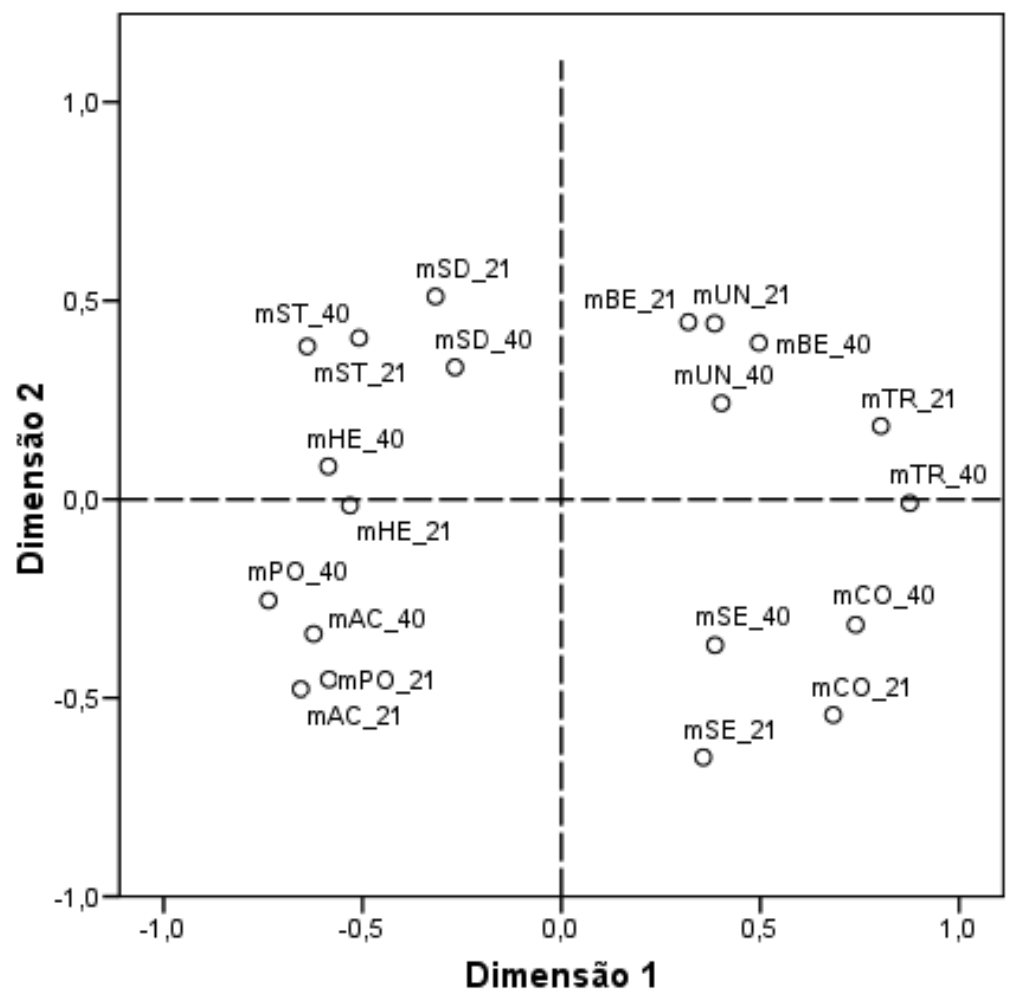

Figura 8. WCMDS (PVQ-40 e PVQ-21).

Assim como nos tratamentos em separado das escalas PVQ-40 e PVQ-21, os valores Universalismo e Benevolência, e ainda Poder e Realização mantiveram trocadas as suas posições.

\section{Considerações Finais}

O objetivo proposto para este artigo foi plenamente alcançado no que se refere à comparação das duas escalas PVQ-40 e PVQ-21, tendo-se ainda validado a estrutura teórica dos valores básicos mediante a escala PVQ-21.

As estruturas encontradas para ambas as escalas refletem a teoria de valores básicos proposta por Schwartz
(1992) em seu modelo original. Ambas as escalas mostraram serem equivalentes quando se trata da estrutura bi-dimensional e bi-polar, opondo corretamente os valores de Autotranscendência versus Autopromoção e os valores de Conservação versus Abertura a Mudança.

Com relação aos tipos motivacionais, ambas as escalas apresentaram as mesmas inversões no que se refere aos valores de Universalismo e Benevolência, assim como para Poder e Realização, inversões já identificadas na literatura, mesmo com a aplicação de outras escalas como a SVS (Bilsky \& Peters, 1999) e CPVC (Bilsky, Brocke, et al., 2008a) em versões de aplicação da MDS exploratória. 
No entanto, quando se trata de comparar a estrutura obtida quanto aos tipos motivacionais, a estrutura difere em relação aos tipos que ocupam as mesmas regiões: enquanto o PVQ-40 agrega na mesma região os valores Estimulação e Autodeterminação, esses valores aparecem em regiões distintas no PVQ-21 e, enquanto no PVQ-21 os valores de Realização e Poder aparecem ocupando a mesma região, no PVQ-40 ocupam regiões distintas. Ambos os instrumentos, porém apresentam uma região comum para os valores Universalismo e Benevolência e distintas regiões para os demais valores.

Os resultados encontrados para o PVQ-40 diferem em parte dos obtidos por Tamayo e Porto (2009), conforme foi mostrado no item anterior. Tendo em vista que o estudo desenvolvido por esses autores aplicou MDS exploratória, enquanto na pesquisa que ora apresentamos, os dados foram tratados mediante WCMDS a partir da matriz de desenho proposta por Bilsky, Gollan, et al. (2008), é de esperar que esta estrutura possa ser identificada em pesquisas futuras envolvendo a aplicação do PVQ-40.

A tradução do PVQ-21 realizada mostrou-se adequada uma vez que foi confirmada a estrutura teórica dos valores básicos mediante a sua aplicação.

A aplicação da WCMDS a partir da matriz de desenho proposta por Bilsky, Gollan, et al. (2008) pode ser útil para a validação da tradução de outras escalas de valores que visem identificar a estrutura de valores básicos de Schwartz, como é o caso da escala CPVC (Bilsky, Brocke, et al., 2008), que ainda não foi traduzida e validada no Brasil.

Os resultados apontam para uma indiferença quanto à escolha entre a escala PVQ-40 e PVQ-21, quando se tratar de analisar a relação entre os quatro pólos das dimensões bipolares, uma vez que ambas apresentam estruturas equivalentes. Esta informação é particularmente importante, quando o objetivo do estudo for relacionar os quatro pólos de valores básicos com indicadores de outros constructos, em que se faz necessária a parcimônia quanto ao número de indicadores como é o caso de tratamentos com equações estruturais. Neste caso, a escolha do PVQ-21 tem como vantagem a economia do tempo de aplicação e de tratamento de dados, além de cansar menos os respondentes.

Este trabalho apresenta como um dos limites a amostra não-probabilística, e o fato de ter sido desenvolvida apenas com um tipo de segmento populacional: estudantes universitários de cursos de Administração de Empresas localizados na cidade de São Paulo. Torna-se necessário o desenvolvimento de outras pesquisas, incluindo outros tipos de amostra, e probabilísticas, contemplando as diversas regiões geográficas brasileiras.

\section{Referências}

Babbie, E. (1999). Survey research methods ( $2^{\text {nd }}$ ed.). Belmont, CA: Wadsworth.

Baslevent, C., \& Kirmanoglu, H. (2008). The role of basic personal values in the voting behavior of Turkish people. Istanbul, Turkey: Istanbul Bilgi University. doi:10.2139/ ssrn.1307031. Retrieved April 08, 2009, from http://ssrn. com/abstract $=1307031$
Bilsky, W., Brocke, M., \& Gollan, T. (2008). Online assessment of value preferences by paired comparison. Paper presented at the XIV European Conference on Personality, Tartu, Estonia.

Bilsky, W., Gollan, T., \& Döring A. (2008). Análise confirmatória de escalonamento multidimensional (EMD) de valores baseada em uma matriz de desenho: Uma nota de pesquisa. In M. L. M. Teixeira (Ed.), Valores humanos \& gestão: Novas perspectivas (pp. 213-221). São Paulo, SP: Serviço Nacional de Aprendizagem Comercial.

Bilsky, W., \& Janik, M. (2010). Investigating value structure: Using theory-based starting configurations in multidimensional scaling (Research note). International Journal of Social Psychology, 25(3), 341-349. doi:10.1174/021347410792675606

Bilsky, W., \& Peters, M. (1999). Estrucutra de los valores y la religiosidad - una investigación comparada realizada en México. Revista Mexicana de Psicologia, 16, 77-88.

Borg, I., Groenen, P. J. F., Jehn, K. A., Bilsky, W., \& Schwartz, S. H. (2011). Embedding the organizational culture profile into Schwartz's Theory of Universals in Values. Journal of Personnel Psychology, 10(1), 1-12. doi:10.1027/1866-5888/ a000028

Borg, I., Groenen, P. J. F., \& Mair, P. (2013). Applied multidimensional scaling. New York: Springer.

Borg, I., \& Staufenbiel, T. (2007). Theorien und Methoden der Skalierung (4. Auflage). Bern, Deutschland: Huber.

Braithwaite, V. A., \& Law, H. G. (1985). Structure of human values: Testing the adequacy of the Rokeach Value Survey. Journal of Personality and Social Psychology, 49, 250-263.

Chang, H. W., \& Lin, G. (2008). Effect of personal values transformation on leadership behaviour. Total Quality Management, 19(1-2), 67-77. doi:10.1080/14783360701601967

Davidov, E., Meuleman, B., Billiet, J., \& Schmidt, P. (2008b). Values and support for immigration: A cross-country comparison. European Sociological Review, 24(5), 583-599. doi:10.1093/esr/jen020

Davidov, E., Schmidt, P., \& Schwartz, S. H. (2008a). Bringing values back in: The adequacy of the European Social Survey to measure values in 20 countries. Public Opinion Quarterly, 72(3), 420-445. doi:10.1093/poq/nfn035

European Social Survey Education Net. (2009). Education net. Retrieved April 08, 2009, from http://essedunet.nsd.uib.no

Gouveia, V. V., Martínez, E., Meira, M., \& Milfont, T. L. (2001). A estrutura e o conteúdo universais dos valores humanos: Análise fatorial confirmatória da tipologia de Schwartz. Estudos de Psicologia (Natal), 6(2), 133-142. doi:10.1590/ S1413-294X2001000200002

Hair, J. F., Jr., Anderson, R. E., \& Black, W. C. (2005). Análise multivariada de dados. Porto Alegre, RS: Bookman.

Kamia, M. (2007). Valores pessoais como antecedentes do comportamento proativo nas organizações (Dissertação de mestrado em Administração de Empresas, Universidade Presbiteriana Mackenzie, São Paulo, SP, Brasil).

Malhorta, N. K. (2001). Pesquisa de marketing: Uma orientação aplicada (3. ed.). Porto Alegre, RS: Bookman.

Porto, J. B. (2005). Mensuração de valores no Brasil. In A. Tamayo \& J. B. Porto (Eds.), Valores e comportamentos nas organizações (pp. 93-119). Petrópolis, RJ: Vozes.

Rokeach, M. (1973). The nature of human values. New York: Free Press.

Rosenberg, S., \& Kim, M. P. (1975). The method of sorting as a data-gathering procedure in multivariate research. Multivariate Behavioral Research, 10, 489-502. doi:10.1207/ s15327906mbr1004_7

Schwartz, S. H. (1992). Universals in the content and structure of 
Sambiase, M. F., Teixeira, M. L. M., Bilsky, W., Araujo, B. F. V. B. \& De Domenico, S. M. R. (2014). Confrontando Estruturas de Valores: Um Estudo Comparativo entre PVQ-40 e PVQ-21.

values: Theoretical advances and empirical tests in 20 countries. Advances in Experimental Social Psychology, 5, 1-65.

Schwartz, S. H. (2005a). Valores humanos básicos: Seu contexto e estrutura intercultural. In A. Tamayo \& J. B. Porto (Eds.) Valores e comportamentos nas organizações (pp. 17-55). Petrópolis, RJ: Vozes.

Schwartz, S. H. (2005b). Validade e aplicabilidade da teoria de valores. In A. Tamayo \& J. B. Porto (Eds.), Valores e comportamentos nas organizações (pp. 56-95). Petrópolis, RJ: Vozes.

Schwartz, S. H. (2006a). Há aspectos universais na estrutura e no conteúdo dos valores humanos? In M. Ros \& V. V. Gouveia, Psicologia social dos valores humanos: Desenvolvimentos teóricos, metodológicos e aplicados (pp. 55-85). São Paulo, SP: Serviço Nacional de Aprendizagem Comercial.

Schwartz, S. H. (2006b). Les valeurs de base de la personne: théorie, mesures et applications. Revue Française de Sociologie, 42, 249-288.

Schwartz, S. H., \& Bilsky, W. (1987). Toward a universal psychological structure of human values. Journal of Personality and Social Psychology, 53(3), 550-562. doi:10.1037/00223514.53.3.550

Schwartz, S. H., \& Sagiv, L. (1995). Identifying culture-specifics in the content and structure of values. Journal of Cross-Cultural Psychology, 26, 92-116. doi:10.1177/0022022195261007

Silva, L. S. S. (2010). Valores organizacionais e pessoais como fatores de impacto no bem-estar ocupacional (Dissertação de mestrado em Administração de Empresas, Universidade Presbiteriana Mackenzie, São Paulo, SP, Brasil).

Tamayo, A. (2007). Contribuições ao estudo dos valores pessoais, laborais e organizacionais [Edição especial]. Psicologia: Teoria e Pesquisa, 23, 17-24. doi:10.1590/S010237722007000500004

Tamayo, A., \& Porto, J. (2009). Validação do Questionário de Perfis de Valores (QPV) no Brasil. Psicologia: Teoria e Pesquisa, 25(3), 369-376. doi:10.1590/S0102-37722009000300010

Tamayo, A., \& Schwartz, S. H. (1993). Estrutura motivacional dos valores humanos. Psicologia: Teoria e Pesquisa, 9(2), 329-348.

Teixeira, M. L. M., \& Monteiro, R. S. (2008). Valores pessoais no ambiente de negócios. In M. L. M. Teixeira (Ed.), Valores humanos \& gestão: Novas perspectivas (pp. 197-211). São Paulo, SP: Serviço Nacional de Aprendizagem Comercial.

Verkasalo, M., Lönnqvist, J. E., Lipsanen, J., \& Helkama, K. (2008). European norms and equations for a two dimensional presentation of values as measured with Schwartz's 21-item portrait values questionnaire. European Journal of Social Psychology, 38, 1-13. doi:10.1002/ejsp.580 